\title{
SOME TYPES OF BOREL MEASURES
}

\section{ROY A. JOHNSON}

1. Introduction. Let $X$ be a locally compact Hausdorff space. Our definitions of Baire sets, Borel sets, Borel measures and regular Borel measures are those of [2]. All measures considered here will be nonnegative Borel measures on $X$, and $\mu, \nu$, and $\lambda$ are reserved for such measures. (Recall Borel measures in [2] are always $\sigma$-finite.) In $\$ 2$ we show that given a Borel measure $\mu$, we may distill from $\mu$ a largest regular measure $\mu_{0}$ such that $\mu=\mu_{0}+\mu_{1}$. The residual measure $\mu_{1}$ will not only fail to be regular; it will be singular with respect to all regular Borel measures on $X$. A Borel measure $\mu$ will be called monogenic if the only Borel measure agreeing with $\mu$ on Baire sets is $\mu$ itself. In $\$ 3$ we show that given a Borel measure $\mu$, we may distill from $\mu$ a largest monogenic measure $\mu_{0}$.

We use the concepts of singularity $(\mu \perp \nu)$ and absolute continuity $(\nu \ll \mu)$ extensively. The definitions are those of [2, pp. 126 and 124]. The following properties will be used implicitly and explicitly:

I. If $\nu \ll \mu$ and $\lambda \perp \mu$, then $\lambda \perp \nu$.

II. If $\nu \ll \mu$ and $\nu \perp \mu$, then $\nu=0$ [2, Exercise 30.9].

III. If $\mu \leqq \nu+\lambda$ and $\mu \perp \lambda$, then $\mu \leqq \nu$.

IV. If $\mu \perp \lambda$ and $\nu \perp \lambda$, then $(\mu+\nu) \perp \lambda$ [2, Exercise 30.10].

V. If $\mu_{n}(E) \rightarrow \mu(E)$ for each Borel set $E$ and if $u_{n} \perp \nu$ for each $n$, then $\mu \perp \nu$.

VI. If (1) $\mu_{\alpha}(E) \uparrow \mu(E)$ for each Borel set $E$, (2) $\mu_{\alpha} \perp \nu$ for each $\alpha$ and (3) $\mu$ or $\nu$ is regular, then $\mu \perp \nu$ [3, 3.1 and 3.3]. Hence, if (1) $\mu=\sum \mu_{\alpha}$ or $\mu=\bigvee_{\mu_{\alpha}}$ (the smallest measure $\geqq$ each $\mu_{\alpha}$ ), (2) $\mu_{\alpha} \perp \nu$ for each $\alpha$ and (3) $\mu$ or $\nu$ is regular, then $\mu \perp \nu$.

VII. Lebesgue decomposition theorem (e.g. $[3,3.4]$ ). Suppose $\mu$ and $\nu$ are Borel measures on $X$ and that $\mu$ or $\nu$ is regular. Then there exist unique Borel measures $\mu_{0}$ and $\mu_{1}$ such that $\mu=\mu_{0}+\mu_{1}$, where $\mu_{0} \ll \nu$ and $\mu_{1} \perp \nu$.

Although properties I-V hold for abstract (not necessarily Borel) measures, properties VI and VII do not always hold, even for $\sigma$-finite measures. (It is understood that "each Borel set $E$ " is replaced by "each measurable set $E$ " in V and VI.) I do not know if the regularity of $\mu$ or $\nu$ is essential in VI and VII.

2. Regular and antiregular measures. If $\mu$ and $\nu$ agree on all Baire

Presented to the Society, August 30, 1968; received by the editors October 9, 1968. 
sets, we say that $\mu$ and $\nu$ are Baire relatives or simply relatives. If $\mu$ is a Borel measure, then its restriction to the Baire sets is a Baire measure which can be extended to a unique regular Borel measure $\mu^{\prime}$ $[2,54 . \mathrm{D}]$. Clearly $\mu^{\prime}$ is a relative of $\mu$, which we may call the regular relative of $\mu$. Of course $\mu=\mu^{\prime}$ if and only if $\mu$ is regular. Finally, we say that $\mu$ is antiregular if and only if $\mu \perp \nu$ for each regular measure $\nu$. Evidently the only measure which is both regular and antiregular is the zero measure.

Our first theorem follows immediately from the definition of antiregular measures and the properties of singularity listed earlier.

THEOREM 2.1. Let $\mathfrak{I}$ be the class of all antiregular Borel measures on $X$. Then:

1. If $\nu \ll \mu$ and $\mu \in \Re$, then $\nu \in \Re$.

2. If $\mu, \nu \in \mathscr{N}$, then so are $\mu+\nu$ and $\mu \mathrm{V}_{\nu}$.

3. If $\mu_{n}(E) \rightarrow \mu(E)$ for each Borel set $E$ and if $\mu_{n} \in \mathcal{N}$ for each $n$, then $\mu \in \Re$.

4. If $\mu_{\alpha} \in \Re$ for each $\alpha$ and if the Borel measure $\mu$ can be written as $\sum \mu_{\alpha}$ or $\bigvee_{\mu_{\alpha}}$ (the smallest measure $\geqq$ each $\mu_{\alpha}$ ), then $\mu \in \Re$.

THEOREM 2.2. If $\mu \perp \mu^{\prime}$, where $\mu^{\prime}$ is the regular relative of $\mu$, then $\mu$ is antiregular.

Proof. Suppose $\nu$ is a regular Borel measure on $X$. Then by the Lebesgue decomposition theorem, we may write $\mu=\mu_{0}+\mu_{1}$, where $\mu_{0} \ll \nu$ and $\mu_{1} \perp \nu$. Then $\mu_{0}$ is regular [2, Exercise 52.9]. Now if $\mu_{1}^{\prime}$ and $\mu^{\prime}$ are the regular relatives of $\mu_{1}$ and $\mu$, respectively, then it is easy to see that $\mu^{\prime}=\mu_{0}+\mu_{1}^{\prime}$, so that $\mu_{0} \leqq \mu^{\prime}$. But since $\mu \perp \mu^{\prime}$, it follows that $\mu_{0}=0$, and we are done.

THEOREM 2.3. If $\mu$ is any Borel measure, then there exist unique regular $\mu_{0}$ and antiregular $\mu_{1}$ such that $\mu=\mu_{0}+\mu_{1}$.

Proof. Let $\mu^{\prime}$ be the regular relative of $\mu$. By the Lebesgue decomposition theorem we have $\mu=\mu_{0}+\mu_{1}$, where $\mu_{0} \ll \mu^{\prime}$ and $\mu_{1} \perp \mu^{\prime}$. Necessarily, $\mu_{0}$ is regular. We show that $\mu_{1}$ is antiregular. For, let $\mu_{1}^{\prime}$ be the regular relative of $\mu_{1}$. It is clear that $\mu^{\prime}=\mu_{0}+\mu_{1}^{\prime}$, so that $\mu_{1}^{\prime} \leqq \mu^{\prime}$. Since $\mu_{1} \perp \mu^{\prime}$, we have $\mu_{1} \perp \mu_{1}^{\prime}$, which shows that $\mu_{1}$ is antiregular.

To prove uniqueness, suppose $\mu=\mu_{2}+\mu_{3}$, where $\mu_{2}$ is regular and $\mu_{3}$ is antiregular. Since $\mu_{0} \leqq \mu$ and $\mu_{0} \perp \mu_{3}$, we have $u_{0} \leqq \mu_{2}$ by property III in the Introduction. Similarly, $\mu_{2} \leqq \mu_{0}$, so that $\mu_{0}=\mu_{2}$ and $\mu_{1}=\mu_{3}$.

TheOREM 2.4. A Borel measure $\mu$ is antiregular if and only if there exists a locally Borel set $A$ (i.e. $E \cap A$ is Borel for each Borel set $E$ ) 
such that $\mu(E-A)=0$ for all Borel sets $E$ and such that $\mu(C)=0$ for each compact set $C \subset A$.

Proor. Suppose $\mu$ is antiregular. Then $\mu \perp \mu^{\prime}$, where $\mu^{\prime}$ is the regular relative of $\mu$. Hence there exists a locally Borel $A$ such that $\mu(E-A)$ $=0=\mu^{\prime}(E \cap A)$ for each Borel set $E$. Now if $C$ is a compact subset of $A$, evidently $\mu^{\prime}(C)=0$. Since there exists a compact $G_{\delta}$ set $D$ such that $\mu^{\prime}(C)=\mu^{\prime}(D)[1,59.1]$, we have $0=\mu^{\prime}(D)=\mu(D) \geqq \mu(C)$, so that $\mu(C)=0$.

On the other hand, suppose there exists such an $A$ as described above. We know that $\mu=\mu_{0}+\mu_{1}$, where $\mu_{0}$ is regular and $\mu_{1}$ is antiregular, so that it suffices to show that $\mu_{0}=0$. Suppose to the contrary that $\mu_{0}(E)>0$ for some Borel set $E$. Then $\mu_{0}(E \cap A)>0$ and by regularity of $\mu_{0}$ there exists a compact $C \subset E \cap A$ such that $\mu_{0}(C)>0$. Of course $\mu(C)>0$ in this case, and that is impossible since $C \subset A$.

THEOREM 2.5. If $\mu$ is antiregular, then $\mu(\{x\})=0$ for each $x \in X$.

Proof. For each $x \in X$, let $\nu_{x}(E)=1$ or 0 according to whether $x \in E$ or $x \notin E$. Then $\nu_{x}$ is a regular measure, so that $\mu \perp \nu_{x}$. It follows that $\mu(\{x\})=0$.

3. Monogenic and antimonogenic measures. We shall say that a Borel measure $\mu$ is monogenic if the only relative of $\mu$ is $\mu$ itself (cf. $[1$, p. 231]). Such a measure is necessarily regular. We shall say that a Borel measure $\mu$ is antimonogenic if $\mu$ is singular with respect to every monogenic measure. Then Theorem 2.1 holds if $\Re$ is taken to be the class of antimonogenic measures.

Evidently the only measure which is both monogenic and antimonogenic is the zero measure. It is also clear that every antiregular measure is antimonogenic. But there are antimonogenic measures which are not antiregular. Indeed, a necessary and sufficient condition for a Borel measure to be antimonogenic is that it have an antiregular relative. We prove the sufficiency now.

THEOREM 3.1. If $\mu$ has an antiregular relative, then $\mu$ is antimonogenic.

Proof. We wish to show that if $\lambda$ is monogenic, then $\mu \perp \lambda$. We consider first the case in which $\mu$ is regular. If $D$ is a Baire set, we define a measure $\mu_{D}$ by $\mu_{D}(E)=\mu(E \cap D)$ for each Borel set $E$. By hypothesis, $\mu$ has an antiregular relative $\nu$, and it is clear that $\mu_{D}$ and $\nu_{D}$ are relatives since $D$ is a Baire set.

Now suppose $D$ is a fixed Baire set. By the Lebesgue decomposition theorem we may write $\lambda=\lambda_{0}+\lambda_{1}$, where $\lambda_{0} \ll \mu_{D}$ and $\lambda_{1} \perp \mu_{D}$. By the 
Radon-Nikodym theorem, there exists a Borel function $f$ such that $\lambda_{0}(E)=\int_{E} f d \mu_{D}$ for each Borel set $E$. Since $\mu_{D}$ is regular, we may assume that $f$ is a Baire function $[1,68.1]$. Define $\lambda_{2}(E)=\int_{E} f d \nu_{D}$ for each Borel set $E$. Since $\lambda_{2} \ll \nu$, we have $\lambda_{2}$ is antiregular. Since $f$ is a Baire function and since $\mu_{D}$ and $\nu_{D}$ are relatives, it follows that $\lambda_{0}$ and $\lambda_{2}$ are relatives $[1,66.1]$. Since $\lambda=\lambda_{0}+\lambda_{1}$ and $\lambda_{2}+\lambda_{1}$ are relatives and since $\lambda$ is monogenic, we have $\lambda=\lambda_{2}+\lambda_{1}$. But since $\lambda$ is regular and $\lambda_{2}$ is antiregular, we have $\lambda=\lambda_{1}$, so that $\lambda \perp \mu_{D}$.

The measures $\mu_{D}$ are increasingly directed in the obvious sense, and $\mu=\mathrm{LUB} \mu_{D}$. Since $\mu_{D} \perp \lambda$ for each Baire set $D$, we have $\mu \perp \lambda$. This completes the case where $\mu$ is regular.

Now suppose $\mu$ is not necessarily regular. Since $\mu$ has an antiregular relative, so does $\mu^{\prime}$, where $\mu^{\prime}$ is the regular relative of $\mu$. Hence $\mu^{\prime}$ is antimonogenic. Now $\mu=\mu_{0}+\mu_{1}$, where $\mu_{0}$ is regular and $\mu_{1}$ is antiregular. Since $\mu_{0} \leqq \mu^{\prime}$ and $\mu^{\prime} \perp \lambda$, whenever $\lambda$ is monogenic, we have $\mu_{0} \perp \lambda$. Of course $\mu_{1} \perp \lambda$, so that $\left(\mu_{0}+\mu_{1}\right) \perp \lambda$, as was to be shown.

LEMMA 3.2. If $\mu$ is not monogenic, then there exists a nonzero $\lambda$ such that $\lambda \leqq \mu$ and such that $\lambda$ has an antiregular relative.

Proof. If $\mu$ is not regular, the conclusion is clear. We may assume $\mu$ is regular. Then $\mu$ has a nonregular relative, $\nu$. We may write $\nu=\nu_{0}+\nu_{1}$, where $\nu_{0}$ is regular and $\nu_{1}$ is antiregular. If $\lambda$ is the regular relative of $\nu_{1}$, then $\nu_{0}+\lambda=\mu$, so that $\lambda$ will serve as the required measure.

Theorem 3.3. If $\mu$ is a Borel measure, then there exist Borel measures $\mu_{0}$ and $\mu_{1}$ such that $\mu_{0}$ is monogenic, $\mu_{1}$ has an antiregular relative (and hence is antimonogenic) and such that $\mu=\mu_{0}+\mu_{1}$. The requirement that $\mu_{0}$ and $\mu_{1}$ be monogenic and antimonogenic, respectively, determines them uniquely.

Proof. If $\mu$ is monogenic, we are done. Otherwise there exists nonzero $\lambda \leqq \mu$ such that $\lambda$ has an antiregular relative. Now let us say that a family of measures $\left\{\mu_{\alpha}\right\}$ is admissible if

(1) $\mu_{\alpha}$ is nonzero for each $\alpha$,

(2) $\mu_{\alpha}$ has an antiregular relative $\nu_{\alpha}$ for each $\alpha$, and

(3) $\sum \mu_{\alpha} \leqq \mu$.

Let us order the (nonempty) collection of admissible families by inclusion. By Zorn's lemma there exists a maximal admissible family, which we label $\left\{\mu_{\alpha}\right\}$. Let $\mu_{1}=\sum \mu_{\alpha}$, and let $\mu_{0}$ be that unique measure such that $\mu=\mu_{0}+\mu_{1}$.

If, for each $\alpha, \nu_{\alpha}$ is an antiregular relative for $\mu_{\alpha}$, then $\sum \nu_{\alpha}$ is easily seen to be an antiregular relative of $\mu_{1}=\sum \mu_{\alpha}$. Also $\mu_{0}$ is monogenic in 
view of Lemma 3.2 and the maximality of the family $\left\{\mu_{\alpha}\right\}$. The uniqueness argument is the same as that in Theorem 2.3, and we omit it.

Theorem 3.4 (Converse of Theorem 3.1). If $\mu$ is antimonogenic, then $\mu$ has an antiregular relative.

PROof. Suppose $\mu$ is antimonogenic. We have $\mu=\mu_{0}+\mu_{1}$, where $\mu_{0}$ is monogenic and $\mu_{1}$ has an antiregular relative. Since $\mu \perp \mu_{0}$, we have $\mu=\mu_{1}$.

THEOREM 3.5. A Borel measure $\mu$ is monogenic if and only if it is singular with respect to every antimonogenic measure.

PROOF. We prove sufficiency. Suppose $\mu$ is singular with respect to every antimonogenic measure. We have $\mu=\mu_{0}+\mu_{1}$, where $\mu_{0}$ is monogenic and $\mu_{1}$ is antimonogenic. Since $\mu \perp \mu_{1}$, we have $\mu=\mu_{0}$.

In view of Theorem 3.5, we see that Theorem 2.1 holds if we take $\Re$ to be the class of monogenic measures.

4. Examples. Let $I$ be any uncountable set, and let $X=2^{I}$ be the space of all functions from $I$ in to the discrete space of two elements, with the product topology. If $x \in X$, then $x$ can be written as $\chi_{B}$, the characteristic function of some $B \subset I$. Now if $x_{0} \in X$ and $\mu$ is the Borel measure on $X$ such that $\mu(E)=1$ or 0 as $x_{0} \in E$ or $x_{0} \notin E$, then $\mu$ is antimonogenic. Indeed, there exist uncountably many mutually singular Borel measures $\nu_{A}$ such that $\nu_{A}\left(\left\{x_{0}\right\}\right)=0$ and such that $\nu_{A}(U)=1$ if $U$ is an open set containing $x_{0}$. Such measures are easily seen to be antiregular relatives of $\mu$.

We show the existence of such measures $\nu_{A}$ for the case $x_{0}=0$. (An obvious translation handles the general case.) Suppose $A$ is an uncountable subset of $I$. Let

$$
S_{A}=\left\{\chi_{B} \in X: B \subset A \text { and } A-B \text { is countable }\right\} .
$$

If $E$ is a Borel set, let $\nu_{A}(E)=1$ if $E \cap S_{A}$ contains a set $F$ such that $F$ is closed with respect to the relative topology on $S_{A}$, and such that given $\chi_{B} \in S_{A}$, there exists $\chi_{C} \in F$ for which $C \subset B$. Otherwise let $\nu_{A}(E)=0$. Using reasoning similar to that of [2, Exercise 52.10], it can be seen that $\nu_{A}$ has the properties described above. Incidentally, $\nu_{A(1)}$ and $\nu_{A(2)}$ are distinct if and only if $A(1) \triangle A(2)$ is uncountable; and when this happens $\nu_{A(1)}$ and $\nu_{A(2)}$ are in fact singular.

Does there exist an antimonogenic Borel measure (or equivalently, an antiregular one) such that each open set has positive measure? The answer is yes, as we now show. Let $I=[0,1]$, and let $X=2^{I}$. Such 
an $X$ is separable, and we let $\left\{x_{n}\right\}$ be a countable dense subset. Define $\mu_{n}(E)=1 / 2^{n}$ or 0 according to whether $x_{n} \in E$ or $x_{n} \notin E$. If $\mu=\sum \mu_{n}$, then $\mu$ is antimonogenic since each $\mu_{n}$ is antimonogenic. Of course, each open set has positive measure since $\left\{x_{n}\right\}$ is dense.

\section{REFERENCES}

1. S. K. Berberian, Measure and integration, Macmillan, New York, 1965.

2. P. R. Halmos, Measure theory, Van Nostrand, New York, 1950.

3. R. A. Johnson, On the Lebesgue decomposition theorem, Proc. Amer. Math. Soc. 18 (1967), 628-632.

Washington State University 